\section{ALL-IN-ONE CEMENT}

seT PP is the syringe version of seT, the all-in-one, radiopaque encapsulated luting cement from SDI. It etches, bonds and fills with just one component.

Containing ionglass, seT PP has been specifically designed to release a

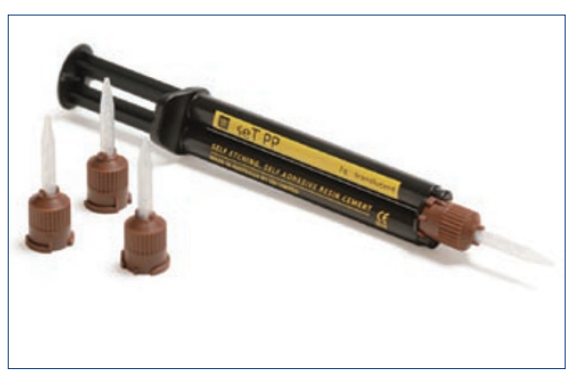

\section{UNIVERSAL GLASS IONOMER}

Light cured glass ionomer Fuji II LC can be used for an array of applications such as final Class III and V restorations, as a base liner beneath composite or amalgam, for all paediatric work and for geriatric work.

Being a true glass ionomer, Fuji II LC will bond chemically to the tooth structure without the need for any separate bonding agents and the material's natural fluoride release is recharged each time a fluoride toothpaste or mouthrinse comes into contact with it. consistently high dose of fluoride over a prolonged period of time. It also minimises post-operative sensitivity as it does not require separate etching and washing. The smear layer remains intact to protect the tooth which reduces sensitivity.

seT PP is a dual cure system: the material hardens in five minutes by itself or in just 20 seconds with the aid of a curing light.

The product is available in five shades and packaged in an introductory kit of five syringes, or refills containing two syringes.

Reader response number 58

Although Fuji II LC is light cured, it will set chemically in the absence of light giving you peace of mind when placing it in very deep or difficult to access areas of the mouth.

There is a choice of presentations with all 11 shades of Fuji II LC. If you choose the hand mix version you can alter the consistency to suit your individual technique. The capsulated version gives you the perfect mix every time and allows you to dispense the material directly from the capsule to the cavity.

Reader response number 59

\section{TAKE CONTROL}

\section{WITH CASSETTES}

Swallow Dental Supplies provide a wide range of cassettes to help dental practices maintain high standards of practice and equipment care that comply with HTM 01-05.

Cassettes can significantly increase protection against injury and cross infection for both patients and the practice team. They can vastly reduce the probability of damage to finely crafted, valuable dental instruments and help introduce an effective quality control system for disinfection, cleaning, sterilisation and storage.

Swallow caters for all cassette needs across all price ranges, such as the MedicalOne, PDT and Stoma cassettes.

Reader response number 60

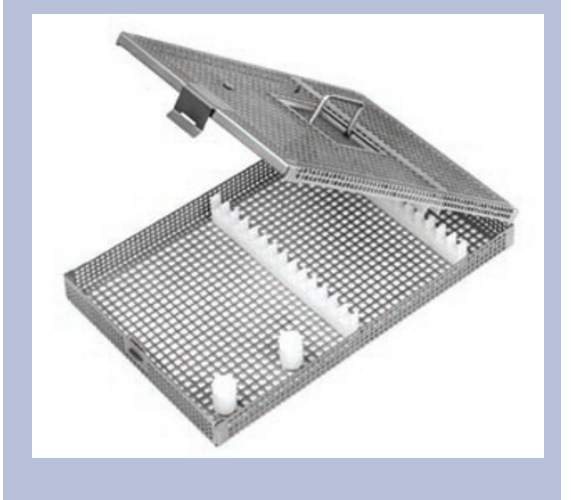

\section{ONE SHADE SIMPLICITY}

Due to the remarkable shade adaptation of GC Gradia Direct to the surrounding tooth structure, you can achieve outstanding aesthetic results even when you only use one shade.

When dealing with more demanding and complex cases a combination of different shades enables unlimited aesthetic results. Gradia Direct offers you the complete assortment from bleach white to dark translucent to outside special shades.

Radiopaque GC Gradia Direct Posterior combines aesthetics with excellent mechanical properties by means of high fracture toughness

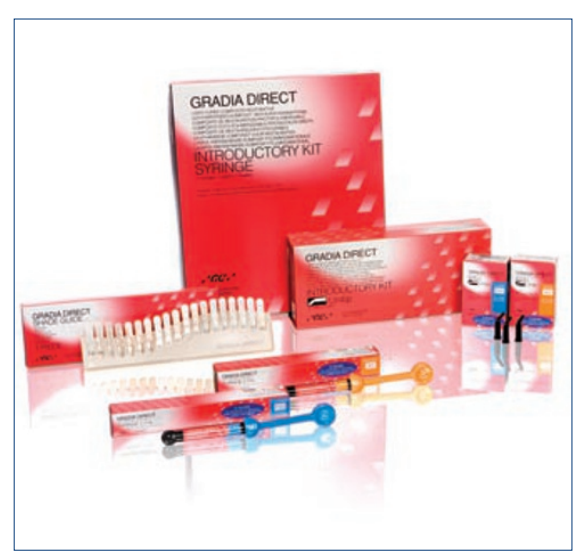

to resist occlusal stress, high wear resistance and low wear on opposing dentition.

GC have developed a new shade guide that takes into account the chroma, hue and value of each composite shade whether you have opted for a single shade or if you are extensively restoring a tooth and require multiple shades. This shade guide incorporates two special shades which are placed underneath or on top of a standard shade to further enhance your restoration.

Reader response number 61 